Article

\title{
Investigation of a Cross-Section with a Constant Transverse Shear Stress Distribution Using a Numerical Approach
}

\author{
Edward T. Bednarz III * (D) and Ryan R. Mulligan \\ Department of Mechanical Engineering, Wilkes University, Wilkes-Barre, PA 18701, USA; \\ ryan.mulligan@wilkes.edu \\ * Correspondence: edward.bednarz@wilkes.edu
}

Received: 28 November 2019; Accepted: 23 December 2019; Published: 24 December 2019

\begin{abstract}
A structural beam which is subjected to shear forces acting perpendicularly to its longitudinal axis will experience longitudinal and transverse shear stresses. In beams where failure in the transverse direction is plausible, it is desirable to maintain a constant transverse shear stress over the beam cross-section to avoid stress concentrations and to use the least amount of material. A numerical approach to the inverse problem of solving for a beam cross-section with a constant transverse shear stress distribution was investigated in this study using Microsoft Excel's Solver and Matlab. The efficiency and shape of the developed cross-section were dependent on the number of elements used to discretize the cross-section. As the number of elements approached infinity, the shape of the cross-section became infinitely thin at the top and infinitely wide at the neutral axis, while also approaching an efficiency of $100 \%$. It is therefore determined that this is an ill-posed inverse problem and no such perfect cross-section exists.
\end{abstract}

Keywords: transverse shear stress; constant stress distribution; structural beam cross-section; inverse problem; ill-posed problem; divergent solution

\section{Introduction}

In the design of a structural beam, there are several geometric and material parameters that must be considered and optimized in order to yield the desired strength, lifespan, and reliability. For typical Euler-Bernoulli beams, the bending stress is much greater than the transverse shear stress. However, there are many cases where the beam is short enough, such that the transverse shear stress dominates. In cases where there is a strong possibility for beam failure in the transverse direction, the shape of a cross-section of a beam can be treated as a design variable. Uniaxial-reinforced composite beams, anisotropic beams, wooden beams, tree branches, bones of young mammals, and railroad ties are all prone to fracture due to their low transverse strength [1-3]. In general, the practical approach to handling high transverse shear stresses is to use reinforcement stiffeners [4-6]. However, there are limitations on the efficiency of these structures. For this methodology, the theoretical solution will be investigated with a perfectly efficient cross-section as the goal.

A structural beam can be subjected to multiple shear forces acting perpendicularly to its longitudinal axis, which could then be reduced to a resultant shear force $V$ at any point along the length of the beam. Longitudinal and transverse shear stresses are developed; the latter of which will be discussed and optimized in this study. The longitudinal shear stress in a beam occurs along the longitudinal axis and is visualized by a slip in the layers of the beam, as shown in Figure 1. The longitudinal shear strength of the beam acts as the agent by which the slip between layers is prevented $[7,8]$. 


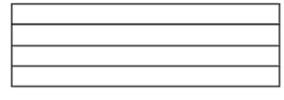

Undeformed State

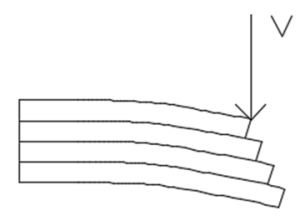

Slip Between Layers

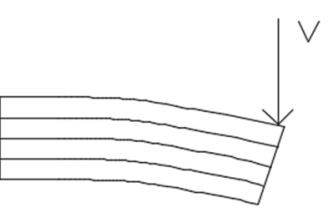

Longitudinal Shear Stress

Figure 1. Longitudinal shear stress in a beam.

The transverse shear stress $\tau$ varies along the cross-section of a beam as shown in Figure 2, where $\tau_{\max }$ is the maximum transverse shear stress and NA is the neutral axis, or the centroid of the cross-section [7]. For the rectangular cross-section shown in Figure 2, the solution is easily derived as a parabolic transverse shear stress distribution which has zero shear stress at the top, peaks at the neutral axis and returns to zero at the bottom [8].

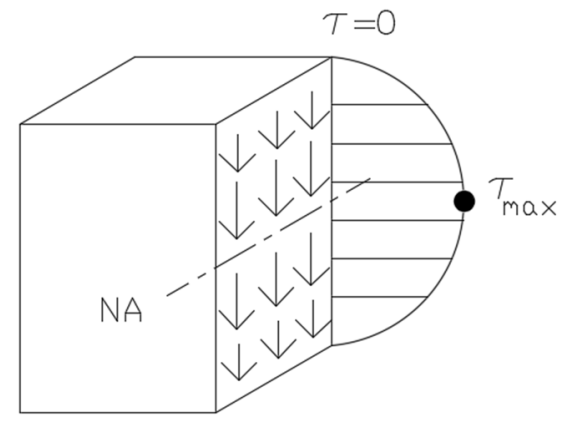

Figure 2. Transverse shear stress distribution for a rectangular cross-section.

The resultant shear force produces longitudinal and transverse shear stresses shown in Figure 3. These stresses must be equal to each other to maintain the equilibrium of an infinitesimal stress element which is located at any theoretical point in the beam [7].

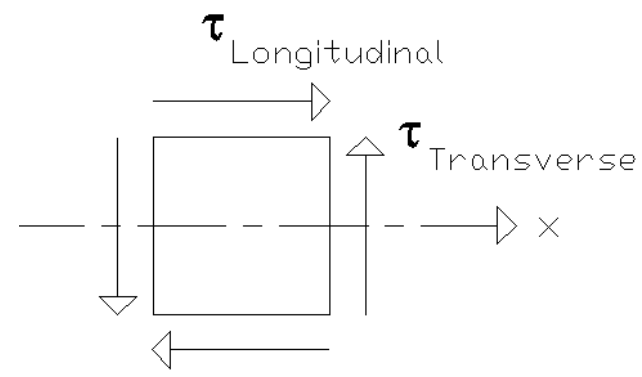

Figure 3. Infinitesimal shear stress element.

It should be noted that $\tau$ is equal to zero at the top and bottom of the beam, shown in Figure 2 . This is due to the fact that the top and bottom boundaries of the beam are exposed to air and therefore carry no longitudinal load [7]. It can also be observed in Figure 2 that the maximum transverse shear stress occurs at the neutral axis. Though this is not always the case, it can be derived that the maximum transverse shear stress will always occur at the neutral axis if the cross-section of the beam is thinnest at that point [7].

For the optimization of the overall lifespan of a beam which may be prone to transverse shear failure, it is desirable to maintain an even transverse shear stress distribution along the cross-section in order to avoid shear stress concentrations within the part. In general, a part is only as strong as its highest stress value. Any material that is not carrying a stress close to that of the maximum is wasted and is therefore unnecessary. Optimization is obtained by altering the geometry of the cross-section.

The optimization of a beam for transverse shear stress efficiency can be solved as an inverse problem. The output (constant transverse shear stress along the cross-section) is known while the input 
(the geometry of the cross-section) is not. The determination of the shape of the cross-section of the beam to achieve maximum overall transverse shear stress efficiency can be completed using general analytical or numerical methods. Both methods are discussed, but only the solution using the latter method is identified and utilized in this study. A numerical method was chosen because of its proven effectiveness in a wide variety of problem-solving and parameter-optimization applications $[9,10]$. Numerical/iterative approaches, such as the use of Microsoft Excel 2013's Solver and MATLAB 8.3, have been used before to solve and verify solutions to similarly-posed problems, such as in the optimization of cylindrical bar cross-sections [10], and open beam geometry [9]. Other approaches using the boundary element method have shown how the transverse shear stress can vary within an arbitrary cross-section [11].

\section{Theory}

\subsection{Transverse Shear Stress Distribution Within a Cross-Section-Analytical Formulation}

For a beam experiencing a resultant shear force $V$ through external loads, a transverse shear stress distribution exists within the cross-section having a height of $h$ as shown in Figure 4 . The cross-section is defined as $z$ (horizontal) as a function of $y$ (vertical) in the first quadrant, with the shaped assumed to be symmetric about both the $y$ and $z$ axes. For the cross-section, three geometric properties can be calculated analytically [7]. The area $A$ is calculated in Equation (1) by setting up a differential element with a height of $d y$ and width of $2 z$. The area of the differential element is $d A=2 z d y$.

$$
A=\int_{\text {Area }} d A
$$

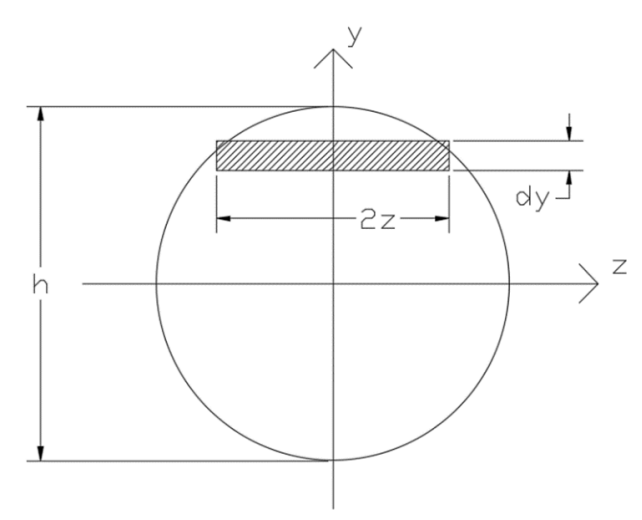

Figure 4. Cross-section of a beam.

By using the symmetry about the $z$ axis, the limits of $y$ are from 0 to $h / 2$ and this value can be doubled. The area will be constant for a given cross-section as shown in Equation (2).

$$
A=4 \int_{0}^{h / 2} z d y
$$

The parameter $Q$ is the first moment of area. The formula is similar to Equation (1) but with the addition of a $y$ term within the integral. $Q$ varies within the cross-section and is calculated from the top down to the point where the stress is formulated for any positive $y$ value as shown in Equation (3).

$$
Q=\int_{\text {Area }} y d A
$$

If the cross-section can be expressed as a simple geometric shape, an alternate formula for $Q$ is used as shown in Equation (4). The portion of the cross-section is drawn from the top down to where 
$Q$ is calculated, $A_{Q}$ is the area and $\bar{y}$ is the centroid. Furthermore, if the portion of the cross-section can be drawn from a series of simple geometric shapes, $Q$ can be expressed from the components of the centroid. In this case, $A_{j}$ is the area of each component and $\overline{y_{j}}$ is its corresponding centroid, where $j$ is an index used for the summation.

$$
Q=\bar{y} A_{Q}=\sum \overline{y_{j}} A_{j}
$$

The last property of the cross-section is the second moment of area, or area moment of inertia $I$. Similar to area, it can also be doubled for symmetry and will be constant for the cross-section as shown in Equations (5) and (6).

$$
\begin{gathered}
I=\int_{\text {Area }} y^{2} d A \\
I=4 \int_{0}^{h / 2} y^{2} z d y
\end{gathered}
$$

The transverse shear stress as a function of vertical position $\tau(y)$ is calculated for any point along the cross-section using the thickness across where the stress is measured $t=2 z$ as calculated in Equation (7) [7].

$$
\tau(y)=\frac{V Q(y)}{I t(y)}
$$

The maximum transverse shear stress can occur at any point along the cross-section. If the thinnest part of the material is at $y=0, \tau_{\max }$ will definitely occur there since $Q$ is at a maximum and $t$ is at a minimum. Otherwise, the derivative of $\tau(y)$ is set equal to zero to find the points where local maxima occur as shown in Equation (8) [12]. Since there could be several local maxima for complex shapes, several points may have to be compared to find the global maximum.

$$
\frac{d \tau}{d y}=0
$$

The average transverse shear stress $\tau_{\text {avg }}$ is calculated in Equation (9) [12].

$$
\tau_{\text {avg }}=\frac{2}{h} \int_{0}^{h / 2} \tau d y
$$

The efficiency of the cross-section $\tau_{\text {eff }}$ is defined in Equation (10). It is expressed as a percentage, with $100 \%$ denoting a constant transverse shear stress distribution.

$$
\tau_{e f f}=\frac{\tau_{a v g}}{\tau_{\max }}(100 \%)
$$

\subsection{Transverse Shear Stress Distribution Within a Cross-Section-Numerical Formulation}

The cross-section from Figure 4 can be discretized into a series of rectangular elements each with a height $H$ as shown in Figure 5. As with the analytical formulation, the shape of the part is assumed to be symmetric about both the $y$ and $z$ axes. The width of an individual element is $2 z_{j}$ where $j$ ranges from 1 to $n$ total elements in the top half. The thickness $t_{j}$ is defined in Equation (11).

$$
t_{j}=2 z_{j}
$$

The numerical solution will approach the analytical solution as the number of iterations increases. If the total number of elements and height of the cross-section are known, the height of each element is calculated in Equation (12).

$$
H=\frac{h}{2 n}
$$


The area $A$ is calculated in Equation (13) by applying Equation (2) to a series of elements, each with an area $A_{j}$.

$$
A=2 \sum_{j=1}^{n} A_{j}=4 H \sum_{j=1}^{n} z_{j}
$$

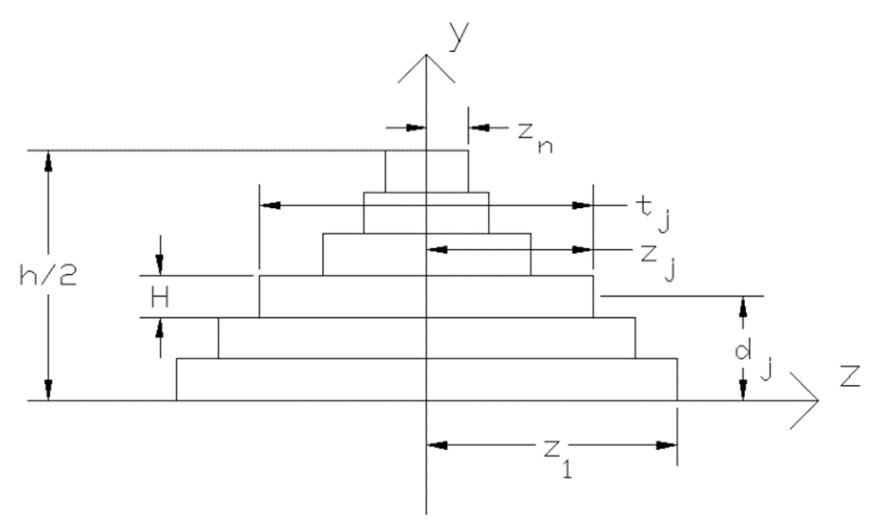

Figure 5. Discretized cross-section.

To calculate the area moment of inertia for each element $I_{j}$, the parallel axis theorem for a given rectangle with width $t_{j}$, height $H$, area $A_{j}$ and distance from the $z$ axis to centroid axis $d_{j}$ as shown in Figure 5, will be applied for each discretized element [7]. The area moment of inertia for each element is $I_{j}$ and the summation for the cross-section is $I$ as shown in Equations (14)-(16).

$$
\begin{gathered}
I_{j}=\frac{t_{j} H^{3}}{12}+A_{j} d_{j}^{2} \\
I_{j}=\frac{z_{j} H^{3}}{6}+2 z_{j} H d_{j}^{2} \\
I=4 H^{3} \sum_{j=1}^{n} z_{j}\left(j^{2}-j+\frac{1}{3}\right)
\end{gathered}
$$

The area $A$ in Equation (13) and inertia $I$ in Equation (16) are constants for the cross-section. The resultant shear force $V$ will also be constant as mentioned previously.

For the numerical method, the index $a$ denotes the vertical position where the stress is calculated. The first moment of area $Q_{a}$ will also depend on where the stress is being calculated as shown in Figure 6 . Using Equation (4) for all the elements from the top down to element $a$ yields $Q_{a}$ in Equation (17):

$$
Q_{a}=H^{2} \sum_{j=a}^{n} z_{j}(2 j-1)
$$

Applying Equation (7) for the numerical method yields the stress $\tau_{a}$ at element $a$ as shown in Equation (18).

$$
\tau_{a}=\frac{V \sum_{j=a}^{n} z_{j}(2 j-1)}{8 H z_{a} \sum_{j=1}^{n} z_{j}\left(j^{2}-j+\frac{1}{3}\right)}
$$

Once all the transverse shear stress values are calculated for the elements, the maximum value is identified. The average stress $\tau_{\text {avg }}$ shown in Equation (19) is the numerical application of Equation (9).

$$
\tau_{a v g}=\frac{\sum_{a=1}^{n} \tau_{a}}{n}
$$


Similar to the analytical method, Equation (10) is applied to the maximum transverse shear stress value that is identified and the average transverse shear stress values from Equation (19) in order to calculate the efficiency of the cross-section.

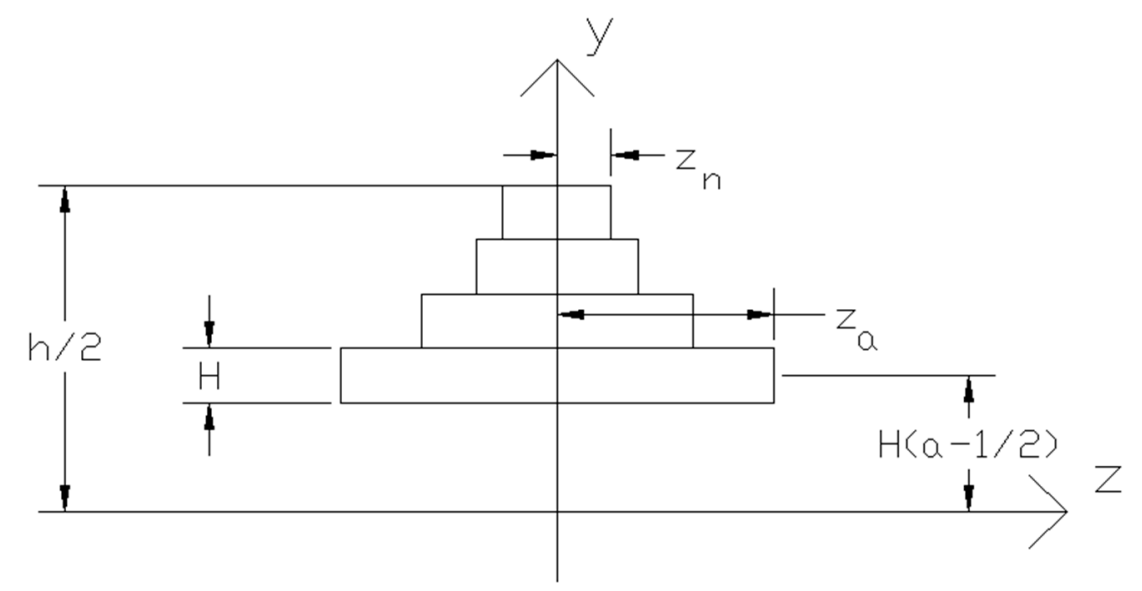

Figure 6. Calculate $Q$ for stress at element $a$.

\subsection{Constant Transverse Shear Stress Distribution}

Using the numerical method presented in Section 2.2, a cross-section is designed in order to produce a constant transverse shear stress distribution. Setting $\tau$ in Equation (7) equal to a constant and recognizing that only two parameters are variable means that $Q$ is proportional to $t$ through a constant $C$ as shown in Equation (20).

$$
Q=C t
$$

Applying Equations (11) and (17) yields an expression for $C$ as shown in Equation (21)

$$
C=\frac{H^{2} \sum_{j=a}^{n} z_{j}\left(j-\frac{1}{2}\right)}{z_{a}}
$$

To determine the constant $C$ for the entire cross-section, $a=n$ is applied to Equation (21), yielding Equation (22).

$$
C=H^{2}\left(n-\frac{1}{2}\right)
$$

Combining Equations (21) and (22) yields Equation (23).

$$
z_{a}=\frac{\sum_{j=a}^{n} z_{j}\left(j-\frac{1}{2}\right)}{n-\frac{1}{2}}
$$

To determine the width of the elements, $a=n-1$ is applied to Equation (23) as shown in Equation (24).

$$
z_{n-1}=z_{n}\left(n-\frac{1}{2}\right)
$$

This means that if the width of the top element $z_{n}$ is predetermined, the next element down $z_{n-1}$ is calculated from it. Furthermore, if this method is repeated, the following pattern emerges in Equations (25)-(27).

$$
\begin{aligned}
& z_{n-2}=\frac{z_{n}\left(n-\frac{1}{2}\right)^{2}}{2} \\
& z_{n-3}=\frac{z_{n}\left(n-\frac{1}{2}\right)^{3}}{6}
\end{aligned}
$$




$$
z_{n-4}=\frac{z_{n}\left(n-\frac{1}{2}\right)^{4}}{24}
$$

This yields a general solution for any element size $z_{j}$ in relation to the top element size $z_{n}$ as shown in Equation (28).

$$
z_{j}=\frac{z_{n}\left(n-\frac{1}{2}\right)^{(n-j)}}{(n-j) !}
$$

From Equation (28) a relationship is developed between the first element $z_{1}$, which is at the neutral axis, and the last element $z_{n}$ as shown in Equation (29).

$$
z_{1}=\frac{z_{n}\left(n-\frac{1}{2}\right)^{(n-1)}}{(n-1) !}
$$

The ratio $z_{R}$ is calculated by dividing the widest element by the narrowest element as shown in Equation (30). For the optimized design, the part will be widest at the neutral axis and thinnest at the top, so it follows from Equation (29) that:

$$
z_{R}=\frac{z_{1}}{z_{n}}=\frac{\left(n-\frac{1}{2}\right)^{(n-1)}}{(n-1) !}
$$

If the width of the neutral axis is held as a constant value, the first element $z_{1}$ is known. Subsequent elements can be derived by combining Equations (28) and (29). Therefore, any element $z_{j}$ can be calculated by the previous element as shown in Equation (31).

$$
z_{j}=\frac{z_{j-1}(n-j+1)}{n-\frac{1}{2}}
$$

The efficiency of the constant transverse shear stress numerical methodology $\tau_{\text {eff }}$ is determined from the fact that the top transverse shear stress element $\tau_{n}$ will always have a value of zero as described previously. However, all remaining transverse shear stress elements $\tau_{a}$ will have the exact same value. Equation (10) is then modified to calculate the efficiency of the cross-section as shown in Equation (32). From Equation (32), as $n$ approaches infinity, $\tau_{\text {eff }}$ approaches $100 \%$.

$$
\tau_{e f f}=\left(\frac{n-1}{n}\right)(100 \%)
$$

\section{Results}

Microsoft Excel and Matlab were both used extensively in this study to model and solve for a beam cross-section which will exhibit a nearly constant transverse shear stress distribution when subjected to a resultant shear force acting perpendicularly to its longitudinal axis. A Matlab script that implemented the equations in Section 2 was developed in order to formulate the above-described cross-section, and Excel's Solver was used to further optimize certain parameters. The methodology derived here is novel and defines the shape of a cross-section in order to obtain a constant transverse shear stress distribution. It will now be investigated if the shape converges to an exact solution as the number of elements $n$ increases.

The constant transverse shear stress solution using the numerical method is compared to the transverse shear stress of a rectangular cross-section in Figure 7. The rectangular cross-section transverse shear stress distribution is a parabola that is derived from Equation (7) and has an efficiency of $66.7 \%$ using Equation (10) [8]. The reason that the rectangular cross-section is not efficient is that the maximum transverse shear stress is well above the average transverse shear stress. The "perfect shape" 
derived here has an efficiency that approaches $100 \%$ as the number of elements approaches infinity from Equation (32).

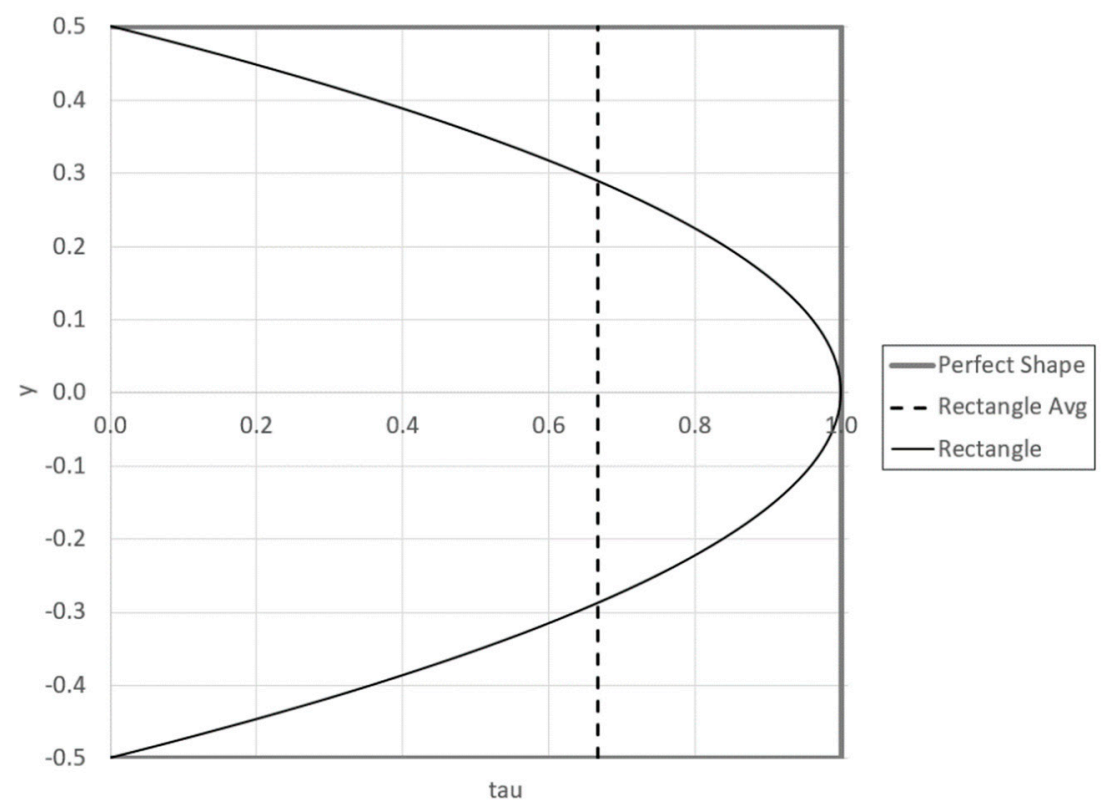

Figure 7. Constant transverse shear stress distribution vs. distribution for a rectangular beam.

The shapes of the cross-sections were dependent on the number of elements chosen. A general observation is that the cross-section is very narrow at the top and extremely wide at the neutral axis. The flare-out point $f_{y}$ is defined as the vertical position on the plot of the cross-section where the shape rapidly becomes much wider. This was defined to be the first $y$ value where the thickness $z$ was calculated to be greater than $0.2 \%$ of $z_{1}$ while moving from the top down.

The produced Matlab script required some user-determined values including $n$ and $z_{1}$. From these values, $z_{n}, z_{R}$, and $\tau_{\text {eff }}$ were then calculated using Equations (30)-(32). The overall width and height were chosen to have a value of one in order to generate a clear-cut plot showing the effect that the chosen $n$ value had on the shape of the cross-section. Starting from $25, n$ was increased by ascending increments to 700 using Matlab, after which the program failed for $z_{1}=0.5$. Excel was used to calculate the shape up to the flare-out point for 4,000,000 elements but could not handle the extremely small values closer to the top of the cross-section. A representation of the cross-sections calculated is shown in Figure 8. As the number of elements increased, the cross-section did not converge on a particular shape. Through extrapolation, the cross-section for an infinite number of elements is infinitely thin at the top, and has a flare-out point approaching $f_{y}=0$.

To bypass the programming constraints, $z_{1}$ was increased to $10^{305}$ (the upper bound for integers in Matlab) and $n$ was increased until the program failed, which was at 1400 elements. For this number of elements, the value of $z_{n}$ was approximately $10^{-305}$ (the lower bound for integers in Matlab.) The log of the $z_{R}$ ratio obtained from each trial was taken to yield a nearly-linear relationship with $n$, as shown in Figure 9. These values were then optimized using Excel's Solver paired with the least squares method to generate an equation and a linear trend line with $R^{2}=1.0000$. The values of $f_{y}$ and $\tau_{\text {eff } f}$ were also recorded for each value of $n$. The data obtained from Matlab and Excel is shown in Table 1.

The linear trend line on the semi-log plot shown in Figure 9 is extrapolated to predict the ratio of the widest to thinnest parts of the cross-section for higher numbers of total elements. It is shown in Table 2 that $z_{R}$ quickly becomes large. With only $n=25$, the ratio is one billion with other larger named numbers shown. 


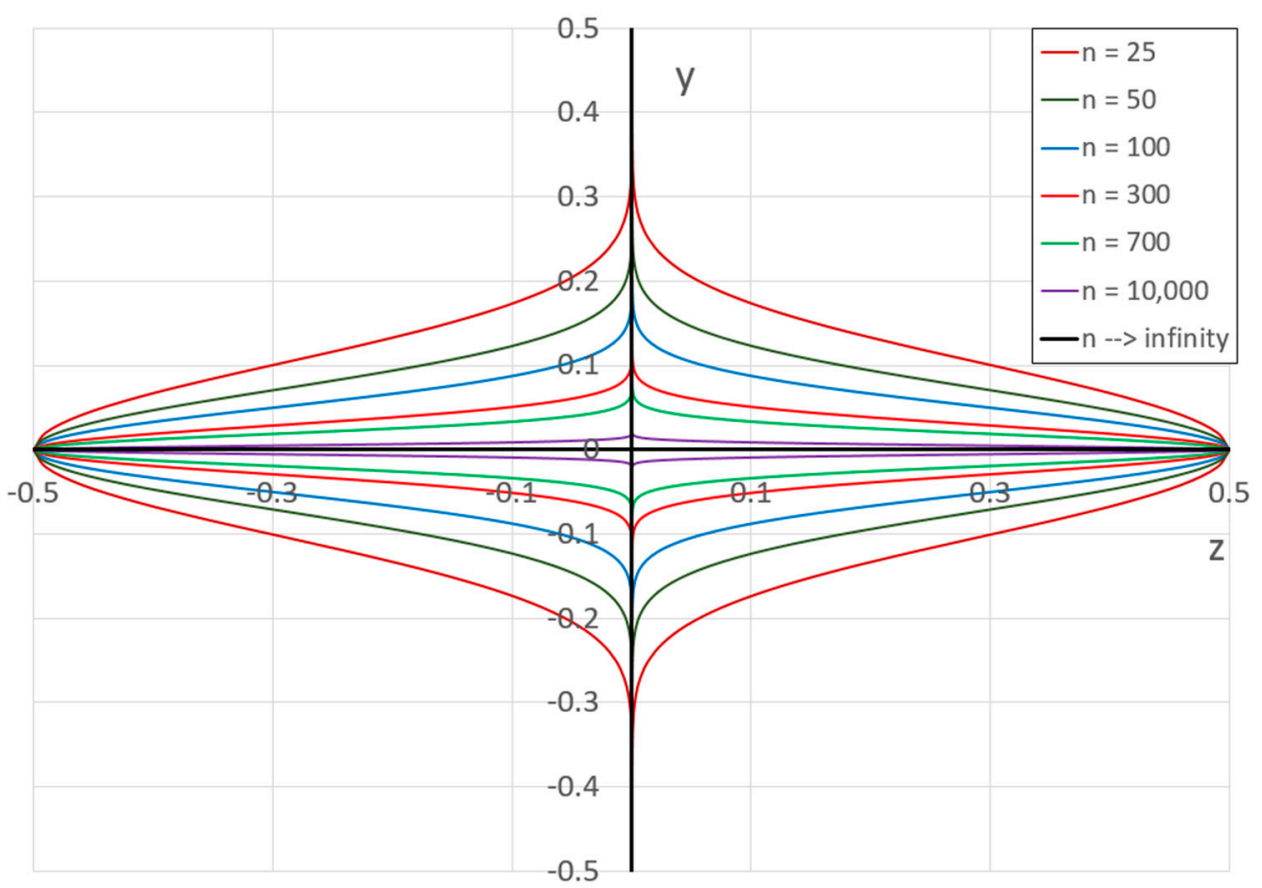

Figure 8. Effect of $n$ on the shape of a cross-section.

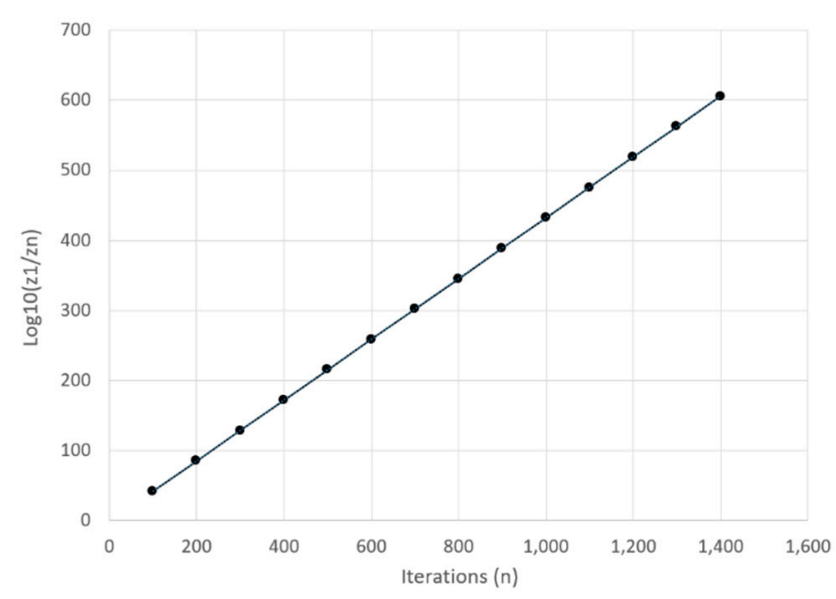

Figure 9. $\log \left(z_{R}\right)$ vs. iterations.

Table 1. Data obtained using Matlab and Excel.

\begin{tabular}{ccccc}
\hline $\boldsymbol{n}$ & $\log \left(z_{R}\right)$ (Calculated in Matlab) & $\log \left(z_{R}\right)$ (Predicted with Solver Formula) & Flare-Out $\left(f_{y}\right)$ & $\tau_{\text {eff }}$ \\
\hline 100 & 41.81 & 43.21 & 0.1717 & $99.00 \%$ \\
200 & 85.09 & 86.43 & 0.1206 & $99.50 \%$ \\
300 & 128.43 & 129.64 & 0.0987 & $99.67 \%$ \\
400 & 171.80 & 172.85 & 0.0865 & $99.75 \%$ \\
500 & 215.18 & 216.07 & 0.0772 & $99.80 \%$ \\
600 & 258.57 & 259.28 & 0.0710 & $99.83 \%$ \\
700 & 301.97 & 302.49 & 0.0658 & $99.86 \%$ \\
800 & 345.37 & 345.71 & 0.0613 & $99.88 \%$ \\
900 & 388.77 & 388.92 & 0.0578 & $99.89 \%$ \\
1000 & 432.18 & 432.13 & 0.0551 & $99.90 \%$ \\
1100 & 475.59 & 475.35 & 0.0523 & $99.91 \%$ \\
1200 & 519.00 & 518.56 & 0.0505 & $99.92 \%$ \\
1300 & 562.41 & 561.77 & 0.0485 & $99.92 \%$ \\
1400 & 605.82 & 604.99 & 0.0465 & $99.93 \%$ \\
\hline
\end{tabular}


Table 2. Extrapolated data.

\begin{tabular}{cccccc}
\hline $\mathbf{n}$ & $\log (\mathbf{n})$ & $z_{\boldsymbol{R}}$ & $\log \left(z_{\boldsymbol{R}}\right)$ & Ratio Name & $\tau_{\text {eff }}$ \\
\hline 7 & 0.84 & 1.0 & 1 & Ten & $85.51706 \%$ \\
9 & 0.96 & 100 & 2 & Hundred & $89.13779 \%$ \\
12 & 1.06 & 1000 & 3 & Thousand & $91.31023 \%$ \\
18 & 1.27 & $1.0 \times 10^{6}$ & 6 & Million & $94.56880 \%$ \\
25 & 1.40 & $1.0 \times 10^{9}$ & 9 & Billion & $96.05011 \%$ \\
32 & 1.51 & $1.0 \times 10^{12}$ & 12 & Trillion & $96.89651 \%$ \\
235 & 2.37 & $1.0 \times 10^{100}$ & 100 & Googol & $99.57403 \%$ \\
702 & 2.85 & $1.0 \times 10^{303}$ & 303 & Centillion & $99.85754 \%$ \\
1400 & 3.15 & $1.0 \times 10^{606}$ & 606 & & $99.92857 \%$ \\
2319 & 3.37 & $1.0 \times 10^{1000}$ & 1000 & & $99.95687 \%$ \\
$2,314,103$ & 6.36 & $1.0 \times 10^{1,000,000}$ & $1.0 \times 10^{6}$ & & $99.99995 \%$ \\
$2.31 \times 10^{9}$ & 9.36 & $1.0 \times 10^{1,000,000,000}$ & $1.0 \times 10^{9}$ & & $99.99999 \%$ \\
\hline
\end{tabular}

\section{Conclusions}

The methodology developed here utilized a numerical solution to solve the inverse problem of determining a structural beam cross-section with a constant transverse shear stress when subjected to a resultant shear force. The cross-section is extremely thin at the top and extremely wide at the neutral axis and does not converge to a specific shape. As the number of elements approaches infinity, the ratio of the widest to the thinnest element will also approach infinity and the point of flare-out approaches the neutral axis. It is therefore determined that this inverse problem is ill-posed.

The cross-section proved to be impractical for use in an engineering application. Due to the divergent nature of the numerical solution, these results cannot be verified through physical experimentation. For future work, this solution can be refined and applied to a more practical beam shape where there are certain constraints in place such as a minimum and maximum thickness to the cross-section. Such a solution would sacrifice efficiency for practicality

Author Contributions: Conceptualization, E.T.B.III; methodology, E.T.B.III and R.R.M.; software, E.T.B.III and R.R.M.; validation, E.T.B.III and R.R.M.; formal analysis, E.T.B.III and R.R.M.; investigation, E.T.B.III and R.R.M.; resources, E.T.B.III and R.R.M.; data curation, E.T.B.III and R.R.M.; writing — original draft preparation, E.T.B.III and R.R.M.; writing - review and editing, E.T.B.III and R.R.M.; visualization, E.T.B.III and R.R.M.; supervision, E.T.B.III; project administration, E.T.B.III. All authors have read and agreed to the published version of the manuscript.

Funding: This research received no external funding.

Acknowledgments: The authors would like to thank Mike Batyko, Corey Smithmyer, and Theologos Tsalis for their contributions to this research.

Conflicts of Interest: The authors declare no conflict of interest.

\section{Nomenclature}

A Area

$A_{j} \quad$ Area of Discrete Element

$A_{Q} \quad$ Area Used to Calculate $Q$

a Numerical Index Where Stress Is Calculated

C Constant Relating $Q$ and $t$

$d_{j} \quad$ Centroid of Discrete Element

$f_{y} \quad$ Flare-out Point

$H \quad$ Height of Discrete Element

h Cross-section Height

I Area Moment of Inertia

$I_{j} \quad$ Area Moment of Inertia of Discrete Element

$j \quad$ Element Index

NA Neutral Axis of Cross-section

$n \quad$ Total Number of Discrete Elements for Top Half of Cross-section 
Q First Moment of Area

$Q_{a} \quad$ First Moment of Area Where Transverse Shear Stress is Calculated

$t \quad$ Sectional Width of Cross-section Where Transverse Shear Stress is Calculated

$t_{j} \quad$ Thickness of Discrete Element

$V \quad$ Shear Force Carried by the Section, Found from the Shear Force Diagram

y Vertical Component of Cross-section

$\bar{y} \quad$ Centroid of Area Used to Calculate $Q$

$\overline{y_{j}} \quad$ Centroid of Component Area Used to Calculate $Q$

$z \quad$ Horizontal Component of Cross-section

$z_{a} \quad$ Half Width of Discrete Element Where Transverse Shear Stress is Calculated

$z_{j} \quad$ Half Width of Discrete Element

$z_{n} \quad$ Half Width of Top Discrete Element

$z_{R} \quad$ Ratio of Widest to Thinnest Discrete Element

$\tau \quad$ Transverse Shear Stress

$\tau_{\text {avg }} \quad$ Average Transverse Shear Stress

$\tau_{a} \quad$ Transverse Shear Stress at Discrete Element $a$

$\tau_{e f f} \quad$ Efficiency of Cross-section

$\tau_{\max } \quad$ Maximum Transverse Shear Stress

\section{References}

1. Ennos, A.R. transverse stresses and modes of failure in tree branches and other beams. Proc. R. Soc. 2009. [CrossRef] [PubMed]

2. Bardella, L.; Tonelli, D. Explicit analytic solutions for the accurate evaluation of the shear stresses in sandwich beams. J. Eng. Mech. 2012, 138, 502-507. [CrossRef]

3. Askarinejad, H.; Dhanasekar, M.; Boyd, P.; Taylor, R. Field Measurement of Wheel-Rail Impact Force at Insulated Rail Joint. In Proceedings of the Society for Experimental Mechanics, Brisbane, Australia, 23 September 2012.

4. Bernardi, P.; Cerioni, R.; Michelini, E.; Sirico, A. Transverse reinforcement optimization of a precast special roof element through an experimental and numerical procedure. Eng. Struct. 2020, 203, 109894. [CrossRef]

5. Jewett, J.; Carstensen, J. Experimental investigation of strut-and-tie layouts in deep RC beams designed with hybrid bi-linear topology optimization. Eng. Struct. 2019, 197, 109322. [CrossRef]

6. Liu, S.; An, X.; Jia, H. Topology optimization of beam cross-section considering warping deformation. Struct. Multidiscip. Optim. 2008, 35, 403-411. [CrossRef]

7. Craig, R. Mechanics of Materials, 3rd ed.; John Wiley and Sons: Hoboken, NJ, USA, 2011.

8. Xia, Y. Transverse Shear in Beams; Lecture Note; The Hong Kong Polytechnic University, Department of Civil Structural Engineering: Hong Kong, China, 2017.

9. Szaroletta William, K. Utilizing Spreadsheet Solver Methods to Determine Optimal Beam Geometry; Purdue University: West Lafayette, Indiana, 2003.

10. Mehne, H. On solving constrained shape optimization problems for finding the optimum shape of a bar cross-section. Appl. Numer. Math. 2007, 58, 1129-1141. [CrossRef]

11. Sapountzakis, E.J.; Mokos, V.G. A BEM solution to transverse shear loading of beams. Comput. Mech. 2005, 36, 384-397. [CrossRef]

12. Finney, R.L.; Thomas, G.B.; Demana, F.; Waits, B.K. Calculus, 1st ed.; Addison-Wesley Publishing: Boston, MA, USA, 1994.

(C) 2019 by the authors. Licensee MDPI, Basel, Switzerland. This article is an open access article distributed under the terms and conditions of the Creative Commons Attribution (CC BY) license (http://creativecommons.org/licenses/by/4.0/). 